\title{
FÍSTULAS DUODENAIS TARDIAS EM TRANSPLANTE PANCREÁTICO COM DRENAGEM VESICAL TRATADAS SEM CONVERSÃO
}

\author{
Late duodenal leaks following pancreas transplantation with bladder \\ drainage treated without conversion
}

João Eduardo Nicoluzzi', Fábio Silveira1, Carlos Marmanillo²

\section{RESUMO}

O uso da drenagem vesical inicialmente aumentou a segurança do transplante pancreático, porém, ao preço de um aumento da morbidade. Fístulas urinárias relacionadas principalmente ao coto duodenal, apesar de pouco freqüentes, ainda são controversas quanto ao tratamento, sendo que muitos defendem a conversão entérica como principal terapêutica. Neste trabalho, apresentam-se dois casos de pacientes com fístulas duodenais tardias, manejados com sucesso sem necessidade de conversão entérica.

Descritores: Fístulas; transplante pancreático; coto duodenal.

\footnotetext{
Instituição: de Misericórdia de Curitiba

\author{
Correspondência: \\ João Eduardo Nicoluzzi \\ R. Santo Amaro, 118 - Água Verde \\ CEP 80620-330 - Curitiba - PR \\ Tel.: $55413264-6719$ \\ E-mail: jenicoluzz@yahoo.com
}

1 Departamento de Cirurgia do Hospital Angelina Caron e da Santa Casa

2 Departamento de Nefrologia da Pontifícia Universidade Católica do Paraná

Recebido em: 18.03.2007
Aceito em: 10.04.2007

\section{INTRODUÇÃO}

O transplante simultâneo pâncreas-rim é um tratamento de longo prazo bem estabelecido para o diabetes mellitus do tipo $1 .{ }^{1} \mathrm{O}$ uso rotineiro de todo o órgão em bloco com o duodeno com drenagem exócrina vesical no final dos anos 80 trouxe melhores resultados e um aumento da aceitação dos transplantes pancreáticos. ${ }^{2}$

As melhorias trazidas pela drenagem vesical na morbi-mortalidade precoce vieram graças a um aumento da morbidade tardia. Essa morbidade relaciona-se com o contato da secreção exócrina pancreática com a bexiga, acarretando algumas complicações (fístulas, cistite, hematúria), que, em alguns casos, necessitam de conversão para a drenagem entérica. ${ }^{3,4}$

Fístulas pós-transplantes permanecem com um importante significado clínico, principalmente pelo fato de serem um importante fator de risco para infecções intra-abdominais. ${ }^{5}$ Uma grande revisão dos enxertos pancreáticos realizados com drenagem vesical identificou que $28 \%$ das fístulas ocorreram nas bordas duodenais. ${ }^{6}$

As fístulas que ocorrem nas bordas duodenais são geralmente tardias (4 semanas pós-transplantes) e causadas por infecção por citomegalovírus ou isquemia, e, na maioria das vezes, requerem conversão para drenagem entérica ${ }^{6,7}$, com elevados custos e morbi-mortalidade.

Neste estudo, demonstramos dois casos de fístula duodenal tardia que foram tratados de maneira bem sucedida de maneira conservadora, sem conversão cirúrgica da drenagem vesical para entérica.

\section{RELATO DO CASO}

A primeira paciente era do sexo feminino, 45 anos de idade, submetida a transplante isolado de pâncreas por diabetes tipo 1 de caráter lábil. Um ano pós-transplante, apresentou quadro clínico 




Figura 1: Fístula (flecha) demonstrada durante cistografia convencional.

de distensão e dor abdominal, associada com elevação dos níveis séricos de amilase. Investigação com tomografia computadorizada demonstrou líquido livre em cavidade abdominal, sem outras alterações significantes. A aspiração guiada revelou que o líquido tratava-se de urina. Uma cistografia convencional (figura 1) confirmou a suspeita clínica de fístula. O tratamento instituído foi a cateterização vesical de demora durante oito semanas. Após esse período, nova cistografia convencional revelou fechamento da fístula (figura 2).

A segunda paciente, feminina, 48 anos de idade, fora submetida a transplante simultâneo pâncreas-rim por diabetes tipo 1. No sétimo mês pós-transplante apresentou semelhante quadro clínico ao previamente descrito. Investigação diagnóstica com tomografia computadorizada abdominal, seguida de aspiração do líquido, revelou novamente a presença de urina.

Foi instituído o mesmo tratamento não cirúrgico, oito semanas de drenagem vesical de demora, dessa vez sem fechamento da fístula. Indicada a cirurgia, procedeu-se a sutura invaginante da borda duodenal seguida de drenagem vesical por duas semanas. A cistografia de controle demonstrou fechamento da fístula.

A captação e o transplante respeitaram os procedimentos padrões. A solução utilizada para captação e armazenamento foi a de Wisconsin, e o tempo médio de isquemia foi de 12 horas.

Ambos os pacientes receberam esquema quádruplo de imunossupressão, somente diferenciada a terapia de indução. A primeira paciente recebeu timoglobulina $(0,5 \mathrm{mg} / \mathrm{kg})$ durante 7 dias. A segunda paciente recebeu basiliximab $20 \mathrm{mg}$ duas horas pré-transplante e a segunda dose no quarto dia pós-transplante. $\mathrm{O}$ restante do esquema constituiu-se de tacrolimus $(0,1 \mathrm{a} 0,2 \mathrm{mg}$ / $\mathrm{kg}$ ) em duas doses, micofenolato mofetil (2g/dia) em 2-4 doses e prednisona. Os níveis de tacrolimus foram mantidos em $8-12 \mathrm{ng} / \mathrm{ml}$ durante os 6 primeiros meses e 5-10ng/ml após esse período.

As pacientes não apresentaram episódios de rejeição ou infecção por citomegalovírus após o tratamento das fístulas. Atualmente se encontram com mais de um ano de seguimento sem recorrência das fístulas.



Figura 2: Cistografia de controle demonstrando fechamento da fístula.

\section{DISCUSSÃO}

As fístulas duodenais nos pós-transplante pancreático são classificadas em precoces ou tardias ${ }^{8}$. Fístulas precoces (menos de 4 semanas pós-transplante) são geralmente da anastomose duodeno vesical, relacionadas a detalhes técnicos e tempos prolongados de isquemia.

As fístulas tardias (mais de 4 semanas pós-transplante), mais frequentemente se originam do enxerto duodenal, causadas por perfuração ou ulceração ${ }^{6,7}$. O processo de ulceração e perfuração pode ser causado por isquemia ou por infecção por citomegalovírus. Outras causas de perfuração incluem complicações de procedimentos invasivos (instrumentação), rejeição e necrose. ${ }^{9}$ Têm sido relatados recentemente alguns fatores de risco, como episódio agudo de rejeição comprovado por biópsia, história de trauma abdominal fechado, episódio recente de infecção por citomegalovírus e uropatia obstrutiva por prostatite aguda. ${ }^{10}$

Tradicionalmente as fístulas precoces são tratadas inicialmente com descompressão vesical por cateterismo de demora, enquanto as fístulas tardias são indicação da conversão da drenagem vesical para drenagem entérica, tratamento da infecção por citomegalovírus, se indicado, e ressecção da área isquêmica duodenal. , $^{7,11}$

Tendo sido observada na literatura a melhora dos sintomas das fístulas tardias com a instalação de cateterismo de demora com cateter de Foley ${ }^{5,6,8}$. A manutenção da cateterização por oito semanas na tentativa de fechamento espontâneo parece lógico ${ }^{12}$, mesmo em casos de fístula tardia. Porém, não devemos ignorar que um tempo prolongado de cateterismo vesical aumenta o risco de infecção urinária, ainda mais em um indivíduo imunodeprimido. ${ }^{13}$

Essa maneira conservadora de tratamento das fístulas tardias evitando a conversão entérica, foi relatada por outros grupos, ${ }^{6,10}$ na busca de se evitar as taxas de complicações da conversão entérica, que se situa na faixa dos $10-20 \% .^{14}$

A taxa de sucesso do manejo conservador (sem conversão) das fístulas tardias varia de $29-64 \%{ }^{6,10}$, e em casos de falha terapêutica (fístula persistente ou deterioração clínica) o reparo cirúrgico é obrigatório. A conversão entérica estaria indicada somente se a fístula é muito grande para ser invaginada e na ausência de infecção urinária ou intra-abdominais. ${ }^{6}$ 


\section{CONCLUSÃO}

Apresentamos dois casos de fístulas duodenais tardias póstransplante de pâncreas, tratados de maneira bem sucedida com descompressão vesical ou reparo direto, evitando a conversão entérica. Acreditamos que esse tipo de tratamento conservador possa ser rotineiramente utilizado, sendo que a conversão entérica deva ser reservada para grandes fístulas quando da ausência de infecção urinária ou intra-abdominal.

\section{ABSTRACT}

Improvements brought by bladder drainage in early postoperative mortality came at the price of late morbidity. Urinary leaks related to duodenal stump, although not very frequent, are still a matter of discussion on the best treatment. Many surgeons support enteric conversion as the best choice. Here, we report two cases of late duodenal leaks successfully managed without enteric conversion.

Keywords: Fistula; Pancreatic Transplantation, Duodenal Stump.

\section{REFERÊNCIAS}

1. Burke GW, Giancio G, Sollinger HW. Advances in pancreas transplantation. Transplantation. 2004;77(9)S62.

2. Sutherland DER, Gruessner RWG, et al. Lessons learned from more than 1000 pancreas transplants at a single institution. Ann Surg. 2001;233(4):463.

3. West M, Gruessner AC, Metrakos P, et al. Conversion from bladder to enteric drainage after pancreaticoduodenal transplantations. Surgery. 1988;124:883.

4. Sollinger HW, Sasaki TM, D'Alessandro AM, et al. Indications for enteric conversion after pancreas transplantation with bladder drainage. Surgery. 1992;112:842.

5. Humar et al. Technical failures after pancreas transplants: Why grafts fail and the risks factors - a multivariate analysis. Transplantation. 2004;78:1188.

6. Hakim NS, Gruessner AC, Papalois BE, et al. Duodenal complications in bladderdrained pancreas transplantation. Surgery. 1997;121:618.

7. Sollinger H, Messing E, Eckhoff D, et al. Urological complications in 210 consecutive simultaneous pancreas-kidney transplants with bladder drainage. Ann Surg. 1993;218:561.
8. Rayhill SC, Odorico JS, Heisey DM, et al. Clinical and laboratory features of pancreatic transplant bladder leaks. Transplant Proc. 1995;27:3141.

9. Gruessner RWG, Dunn DL, Tzardis PJ, et al. Complications occurring after whole organ duodenopancreatic transplantation: relation to the allograft duodenal segment. Transplant Proc. 1990;22:578.

10. Nath DS, Gruessner A, Kandaswamy R, et al. Late anastomotic leaks in pancreas transplant recipients - clinical characteristics and predisposing factors. Clin Transplant. 2005;Apr;19(2):220.

11. Kaplan AJ, Valente JF, First MR, et al. Early operative intervention for urologic complications of kidney-pancreas transplantation. World J Surg. 1998;22:890.

12. Elkhammas EA, Henry ML, Tesi RJ, et al. Late urine leaks after combined kidneypancreas transplantation. Transplant Proc. 1994;26:453.

13. Van der Pijl JW, Smets YFC, de la Fuente R, et al. Urologic infections and problems after 50 simultaneous pancreas-kidney transplantations. Transplant Proc. 1995;27:3105.

14. West M, Gruessner AC, Metrakos P, et al. Surgical complications 